\title{
Le pétrarquisme pieux: la conversion de la poésie amoureuse chez Jean de La Ceppède
}

\author{
ROBERT MELANÇON
}

Dans plusieurs anthologies plus ou moins récentes de la poésie française de la fin du $16^{\mathrm{e}}$ siècle, on peut lire les textes autrement que dans la succession des productions d'un même auteur, selon des regroupements qui répondent à des affinités thématiques ou formelles. ${ }^{1}$ Dans de telles anthologies, la personnalité des divers écrivains - qu'elle reste relativement effacée dans le cas de bien des minores, ou qu'elle s'affirme plus vivement chez un d'Aubigné ou un Sponde - passe au second plan au profit de grandes constantes qui traversent tous les textes. Ce qui s'y donne à lire, c'est ce qu'on pourrait appeler les énoncés poétiques de l'époque, ces grands réseaux de tropes et de topoï qui courent d'une œuvre à l'autre et qui nous invitent à considérer les poèmes comme la production d'une sorte d'archipoète anonyme (ou bien, ce qui revient au même, doté de multiples signatures interchangeables) plutôt que comme les œuvres distinctes d'auteurs individuels. Il faut noter que l'érudition moderne, en dispersant les textes d'un auteur sous les différentes rubriques d'une anthologie, retrouve une pratique ancienne. Les recueils collectifs, qui sont, à la fin du $16^{\mathrm{e}}$ siècle et au début du $17^{\mathrm{e}}$ un des modes essentiels de publication de la poésie-sinon le plus important - ne regroupent pour ainsi dire jamais les textes par auteurs mais selon des classements où la notion même d'auteur individuel tend à se dissoudre. Qu'il s'agisse, par exemple, des Muses R'Alliées de diverses parts de l'éditeur Mathieu Guillemot (1603) ou du Parnasse des plus excellens poetes de ce temps (1607) ou des Delices de la poesie françoise (1620) rassemblés par Toussainct Du Bray, les recueils collectifs ignorent décidément les notions de poète et d'œuvre individuels. Les textes, regroupés par matières, par formes, ou par destinataires, y restent même souvent anonymes, et il a fallu l'immense enquête de Frédéric Lachèvre au début du siècle pour restituer à leurs auteurs certains de ces poèmes, sonnets, odes et stances sur les yeux, les cheveux ou la couleur grise, que rien ne semble devoir distinguer. ${ }^{2}$

Cette pratique éditoriale, qui s'explique vraisemblablement par des 
impératifs commerciaux aux $16^{\mathrm{e}}$ et $17^{\mathrm{e}}$ siècles et qui répond à des objectifs tantôt scientifiques et tantôt pédagogiques pour les anthologies modernes, met en évidence quelques traits essentiels de la poésie de cette époque. D'abord que notre idée d'originalité littéraire n'y a pas cours; ensuite que notre notion d'auteur s'y transpose malaisément et qu'elle se rapproche plus à l'époque du sens étymologique d'augmentateur que de l'idée romantique de créateur; enfin qu'il existe un langage poétique doté de certains caractères spécifiques, que tous les poètes pratiquent et qui constitue une ressource commune dans laquelle chacun puise librement. On pourrait dire de l'écriture poétique qu'elle appartient à la fin du $16^{\circ}$ siècle au domaine public.

La poésie de cette époque est en effet traversée, ou plutôt organisée, structurée par des codes qui la constituent en domaine autonome: systèmes de conventions qui régissent, par exemple, les bonnes manières littéraires de l'éloge de la beauté féminine, réseaux de figures empruntées aux poètes gréco-latins, italiens et à leurs imitateurs, lieux communs et formules si souvent utilisés qu'ils offrent au lecteur le plaisir supplémentaire de la familiarité. ${ }^{3}$ L'existence de ces codes est attestée non seulement par les textes eux-mêmes, mais aussi par divers ouvrages, le plus souvent des usuels, qui en répertorient de façon plus ou moins exhaustive les éléments. Ainsi, les Épithètes de Maurice de La Porte, publiées à Paris en 1571 et qui ne sont rien de moins qu'un dictionnaire du vocabulaire poétique à l'intention des lecteurs (l' "Advertissement" prévient que ce livre est "faict principalement pour l'intelligence des poètes", dont il commente "plusieurs noms propres poétiques avec leur fabuleuse explication"), à l'intention également des poètes eux-mêmes et des auteurs en général puisqu'il s'annonce en soustitre comme "un livre non seulement utile à ceux qui font profession de la poésie, mais fort propre aussi pour illustrer toute autre composition françoise".. ${ }^{4} \mathrm{D}$ 'autres ouvrages, nombreux, remplissent des fonctions analogues: recueils de lieux-communs, florilèges et polyantheae, topiques qui guident l'invention, recueils de citations prêtes à enchasser comme des joyaux dans des contextes inédits, listes d'ornements divers jugés propres à illustrer tout texte..$^{5}$ Jamais peut-être la poésie n'a été autant codifiée qu'à cette époque, riche de conventions qui sont autant des stimulants que des chaînes. Aucun genre ne leur échappe. Elles peuvent être plus immédiatement repérables dans d'innombrables sonnets amoureux, avatars d'un pétrarquisme que le lecteur d'un gros recueil collectif comme l'Academie des modernes poetes françois d'Antoine Du Breuil (1599) se surprend à souhaiter moins surabondant, ou encore dans ces odes officielles dont chaque événement du règne provoque la multiplication; elles n'en informent pas moins tous les genres, y compris le lyrisme religieux. La superposition de quelques vers écrits par divers auteurs permet de constater empiriquement leur emprise. 
Considérons d'abord quelques poèmes sur le thème des yeux - mais thème est ici un mot bien trop vague puisqu'il s'agit d'une topique particulièrement féconde, on dirait d'une véritable machine à produire des vers:

Oeil mon petit mignon, ma douce friandise,

Oeil bien caractéré des mignardes amours,

Oeil miroir de mon coeur...

... Et cet oeil qui me blesse

Ce bel astre besson clairement étoilé ...

Vous n'estes pas de yeux, vous avez trop de flame, Et les yeux des mortels n'avivent point les morts, Vous n'estes pas Soleils, car vous bruslez mon ame, Et les rais du Soleil ne bruslent que des corps.

Ce ne sont pas des yeux, ce sont plustost des Dieux, Ils ont dessus les Rois la puissance absolue;

Dieux non, ce sont des Cieux, ils ont la couleur bleue

Et le mouvement prompt comme celui des Cieux.

Beaux yeux, ardans Amours, clairs Soleils, doux Esprits, Quand verray-je vos traits, vos feux, vos jours, vos flames?

O beaux yeux, beaux Soleils, ô beautez ravissantes, Qui passez de bien loing les beautez de ces lieux, Qui donnez estre, et lustre à la beauté des Cieux, Qui pourroist soustenir vos clartez doux-forçantes?

Puis, élargissant le propos, quelques éloges de la beauté physique, des blasons revus et repolis par des centaines de poètes:

Ce teint vermeil, le séjour de mes yeux,

$\mathrm{Ce}$ front poli de blancheur ivoirine,

Ce beau coral de bouche cinabrine,

Ces doux appas d'un souris gracieux,

Ces deux flambeaux où s'appuie mon mieux ...

Un poil frisé de l'Aurore argentée,

Un arc d'amour, un front semé de lys,

Deux beaux soleils par Phoebus embellis, 


\section{8 / Renaissance and Reformation}

Un rang de perle, un coral, un rubis,

(...)

Un doux attrait où l'âme est arrêtée...

Beaux noeuds crespes et blonds, nonchalamment espars,

(...)

Front de marbre vivant, table claire et polie,

(...)

Yeux pleuvant à la fois tant d'aise et de martyre;

Sous-ris, par qui l'Amour entretient son empire...

Changeant de registre, toujours dans le blason:

Elle a la bouche et l'oeil d'une chate malade,

L'auguste majesté d'une vieille salade;

Sa petite personne et son corps de brochet

Ressemble un trébuchet.

Ces yeux (tantost si beaux), rébattus, r'enfoncez,

Ressalis, sont hélas! deux Soleils éclipséz,

Le coral de sa bouche est ores jaune-pasle.

Les roses, et les lys de son teint sont flétris:

Le reste de son Corps est de couleur d'Opale

Tant de la teste aux pieds ses membres sont meurtris

À dessein je n'ai pas identifié les auteurs de ces vers pour que la mention de divers noms ne vienne pas distraire l'attention de ce langage anonyme qui est, pour l'instant, mon objet. Ce sont, pour les poèmes sur les yeux: Scalion de Virbluneau, Pierre Cornu, François Ménard, Laugier de Porchères, Pierre de Deimier, Jean de La Ceppède; pour les blasons: Claude de Pontoux, Joachim Blanchon, Philippe Desportes, Sigogne, Jean de La Ceppède. ${ }^{6}$

Ces deux listes se terminent par le nom de Jean de La Ceppède, l'auteur des Théorèmes sur le sacré mystère de nostre rédemption. Cela signifie que les derniers vers de chaque série d'exemples appartiennent à un recueil de poésie religieuse tout entier consacré à la méditation de la Passion du Christ. Or, ils ne détonnent pas au milieu des textes parmi lesquels je les ai cités, et leur langage s'intègre au contraire parfaitement, sans la moindre solution de continuité, à des métaphores tirées de poèmes d'amour profanes.

C'est que le code pétrarquiste envahit aussi cette poésie pieuse dont l'oeuvre de La Ceppède propose peut-être l'exemple le plus achevé à la fin de la Renaissance. Toutefois, si nous les replaçons dans leur contexte, si 
nous lisons intégralement les sonnets dont je n'ai jusqu'ici cité que des extraits, il devient aussitôt évident que ce pétrarquisme d'apparence si profane s'intègre parfaitement à la méditation religieuse. Plus exactement on dirait que le langage de la poésie amoureuse se trouve tout à fait christianisé par La Ceppède. Le premier fragment, si étrangement proche de tant de poèmes pétrarquistes sur l'oeillade foudroyante de la dame, provient d'un sonnet sur la puissance du regard du Christ:

Ce ne sont pas sans plus ses paroles puissantes, Qui courbent ces mutins: il sort de ses beaux yeux, Tant d'éclats, tant d'éclairs, que ces meschans Hebrieux Ne peuvent soustenir leur flammes terrassantes,

O beaux yeux, beaux Soleils, ô beautez ravissantes, Qui passez de bien loing les beautez de ces lieux, Qui donnez estre, et lustre à la beauté des Cieux, Qui pourroit soustenir vos clartez doux-forçantes?

Moyse apres avoir à l'Eternel parlé

Fut contraint de tenir son visage voilé, Pour n'esblouir les yeux du peuple Israelite.

La face de mon Christ, brilloit bien autrement. Vous en fistes la preuve, ô peintre Abigarite, Lors de son jour frappa vos yeux d'aveuglement. (I.I.LI)

Le second est tiré d'un ecce homo proprement stupéfiant, envahi par les topoï de la beauté féminine, où la piété se teinte d'un érotisme prodigieux, et qui semblait proche tantôt, dans l'inversion des signe de l'éloge, du grotesque satyrique d'un Sigogne:?

Voicy-l'Homme, ô mes yeux, quel objet déplorable.

La honte, le veiller, la faute d'aliment,

Les douleurs, et le sang perdu si largement

L'ont bien tant déformé qu'il n'est plus desirable.

Ces cheveux ('ornement de son chef venerable)

Sanglantez, herissez, par ce couronnement,

Embrouillez dans ces joncs, servent indignement

A son test ulceré d'une haye execrable.

Ces yeux (tantost si beaux) rébatus, r'enfoncez,

Ressalis, sont helas! deux Soleils éclipsez,

Le coral de sa bouche est ores jaune-pasle.

Les roses, et les lys de son teint sont flétris:

Le reste de son Corps est de couleur d'Opale, 
Tant de la teste aux pieds ses membres sont meurtris.

(I.II.70)

Il convient donc de situer le texte des Théorèmes à l'intérieur d'un espace de discours hors duquel il reste difficilement intelligible: la poésie amoureuse pétrarquiste. Cette mise en situation s'avère d'autant plus nécessaire que La Ceppède a lui-même entouré ses poèmes d'un énorme commentaire dans lequel il cite surabondamment - l'index compte 171 noms - à peu près tous les textes qui peuvent faire autorité en matière de théologie, mais dans lequel il tait à peu près complètement ses sources littéraires. Je ne donnerai qu'un exemple de ces silences. Le premier sonnet reprend l'incipit fameux de l'Énéide: "Arma virumque cano". Or la note qui s'accroche à ce vers, et qui contient près d'une page serrée de texte, ne mentionne pas Virgile. On objecterait à juste titre que La Ceppède n'a vraisemblablement pas cru nécessaire de souligner ce qui était évident pour des lecteurs nourris de littérature classique. Toutefois, dans bien d'autres cas, ces silences portent à conséquence puisque des pans entiers de l'intertexte des Théorèmes restent négligés par les commentateurs modernes, ce qui nous interdit de comprendre à certains égards la portée considérable de ces poèmes.

Sans décrire tout ce réseau on peut s'attacher à définir un des éléments essentiels de l'intertexte des Théorèmes. Cette oeuvre a paru en 1613 et 1622 , mais elle a vraisemblablement commencé d'être écrite vers 1570 , et son noyau primitif avait été publié dès 1594 sous la forme de Douze méditations surle sacré mystère de nostre rédemption. ${ }^{8} \mathrm{Je}$ m'en tiendrai ici au livre de 1613 , qui forme un tout en lui-même.

\section{***}

L'organisation même des Théorèmes sur le sacré mystère de nostre rédemption, un des recueils les plus complexes de toute la poésie française, met en lumière un vaste intertexte. La première partie se divise en trois livres comptant chacun cent sonnets. Ceux-ci sont précédés d'une dédicace à la Reine et d'un substantiel avant-propos qui explique le dessein de l'ouvrage; puis, chaque livre est lui-même précédé d'un argument en prose qui recompose synthétiquement les récits évangéliques de la Passion du Christ, et chaque sonnet est longuement annoté par La Ceppède luimême. Une des notes du sonnet XXXVII du livre I, par exemple, entreprend d'établir en vingt-cinq pages le caractère naturel de la sueur de sang du Christ durant l'agonie au Mont des Oliviers, en alléguant une véritable encyclopédie: les Pères de l'Église, des philosophes, des médecins. À la fin de l'ouvrage, une "table des sonnets" présente pour chacun un sommaire de quelques lignes - il y faut trente-quatre pages - ; elle est suivie d'un index 
des "auteurs de diverse profession alléguez en cet œuvre" qui relève 171 noms: des Pères de l'Église, des Théologiens et des Papes, bien sûr, mais aussi des auteurs profanes de l'Antiquité - philosophes, poètes, médecins, géographes - des humanistes, et jusqu'à un certain Ronsardus allégué plus en tant qu'auteur de "l'Hercule chrestien" qu'en tant que chantre des Amours de Cassandre, de Marie, d'Hélène. Enfin, une "table alphabétique très ample des points principaux et matières plus remarquables traitées en cet œuvre" - nous dirions un index - répertorie en trente-huit pages serrées sur deux colonnes, de "Age de Jésus-Christ en ce monde" à "Zèle", une série de questions théologiques, philosophiques et scientifiques qui font la matière de ces poèmes. Ceux-ci se présentent donc entourés de tout un appareil de commentaires en prose. La Ceppède lui assigne un rôle précis, le protéger d'une éventuelle accusation d'hérésie:

Or pour ce que la calomnie, qui mord ordinairement sur nos actions, attaque principalement les œuvres qui traitent de la religion, pour les arguer d'hérésie, j'ai voulu moy-mesme faire des annotations à mes théorèmes, pour marquer ces lieux de l'Escriture ou des Saincts Pères, qui me doivent estre à garent de tout ce que j'ay escrit. ${ }^{9}$

Rôle modeste, quoique essentiel à une époque où l'accusation d'hérésie portait à fâcheuses conséquences, souligné par le mot discret d'“annotations". ${ }^{10}$ En fait, les notes de La Ceppède outrepassent souvent le rôle qui leur est expressément assigné: elles se gonflent, se détachent des poèmes qu'elles sont censées éclairer et font le tour d'une question en plusieurs pages de prose. Autant qu'un recueil de sonnets, les Théorèmes forment à certains égards un livre d'essais, tant le commentaire prolifère par moments jusqu'à faire oublier les poèmes qui en sont le point de départ et, en principe, la raison d'être. Cet aspect de l'oeuvre mériterait une étude approfondie;"11 en l'attendant, je suggérerai seulement que peu d'œuvres permettent de mieux observer ce genre si déconcertant pour nous - si discrédité dès le $17^{\mathrm{e}}$ siècle - du commentaire humaniste: les poèmes euxmêmes ne se proposent-ils pas comme des amplifications et des commentaires des récits évangéliques de la Passion?

D'où un premier réseau intertextuel dans lequel les Théorèmes prennent place: cette vaste littérature critique, si riche à la fin de la Renaissance. ${ }^{12}$ À cela s'ajoute toute une littérature de dévotion, manuels de prière, traités et recueils de méditation, ${ }^{13}$ ainsi que, bien évidemment, le genre même du recueil de sonnets, avec le grand modèle pétra rquien du Canzoniere, revu et transmis par les poètes de la Pléiade et leurs imitateurs avant d'être corrigé par les nombreux sonnettistes chrétiens de la fin du $16^{\mathrm{e}}$ siècle et du début du 17e: Sponde, Chassignet, Favre, Lazare de Selve.

Canzoniere spirituel, les Théorèmes de La Ceppède renvoient évidemment à 
l'immense corpus pétrarquiste dont ils reprennent la forme et les procédés qu'ils déplacent et réordonnent à d'autre fins que d'amour profane. "L'avant-propos à la France" que La Ceppède place en tête de son œuvre propose une vue cavalière de l'histoire de la poésie française qui éclaire singulièrement l'entreprise de sa poésie sacrée. Ce texte évoque par moments l'Abbregé de l'art poétique françois dont l'ouverture esquisse à grands traits un tableau historique d'une ampleur peu commune, qui conduit de cette "Théologie allégorique" qu'était la poésie aux premiers âges et de ses maîtres mythiques "Eumolpe Cecropien, Line, maistre d'Hercule, Orphée..." appelés "poètes divins (...) pour la conversation qu'ils avoient avec les oracles, Prophètes, Devins, Sybilles, interprètes de songes", aux "poètes humains (...) plus enflés d'artifice et de labeur que de divinitez". ${ }^{14} \mathrm{La}$ Ceppède établit lui aussi la dignité antique ainsi que le caractère sacré de la poésie, en s'appuyant toutefois non seulement sur la tradition classique gréco-latine, mais aussi sur la poésie biblique comme en font foi des références marginales à "David en ses Pseaumes, Moyse, Judith, d'Elborah et autres en leurs cantiq. comme en Exode 15 verset 2, Judith dernier, et autres lieux". ${ }^{15}$ La relation entre l'Antiquité païenne et la Bible n'est pas de simple équivalence entre deux traditions parallèles, prestigieuses chacune dans leur ordre; lorsqu'il arrive à l'Écriture sainte, $\mathrm{La}$ Ceppède note bien qu'il s'agit, dans la perspective qui est la sienne, de "passer des ombres à la lumière":

Le fabuleux crayon des vieilles peintures parlantes nous a pourtrait cette vérité en mille et mille tableaux; tantost figurant cette deesse naissante de Juppien et de Mnémosyne, tantost l'honorant de neuf divers noms tous expressifs de ses diverses perfections; tantost la montrant sur l'Olympe; et tantost la faisant l'agréable compagne du bel Apollon. L'irréprochable témoignage de ceux qu'on estime avoir divinement philosophé appelle les esprits de la poésie sacrez et divins. Les sacrez honneurs, qu'elle a depuis tant de siècles immortalisé, nous témoignent l'immortalité de ses grâces: et l'Eternel (pour passer des ombres à la lumière) nous fit jadis bien voir clairement combien il agréait cette belle, lui faisant ores prononcer les arrests de sa volonté, ores entonner ses louanges; ores rechanter ses bienfaits; ores croniquer la vie et les gestes de son bien-aymé. ${ }^{16}$

Texte essentiel qui définit la poésie ("peinture parlante"), décrit son origine sacrée et lui assigne ses fins en quelques lignes où s'ébauche tout un système des genres: poésie didactique ("prononcer les arrests de la volonté" de l'Éternel), lyrique ("ores entonner ses louanges, ores rechanter ses bienfaits"), épique ("ores croniquer la vie et les gestes de son bien-aymé"). Cette dernière notation porte à conséquence dans la mesure où elle décrit le projet même des Théorèmes: ce canzoniere est aussi une épopée comme le marque expressément l'incipit du premier sonnet dans l'allusion à l'incipit le plus fameux de la poésie latine, véritable embrayeur obligé pour tout poème épique: 
Je chante les amours, les armes, la victoire

Du Ciel, qui pour la Terre a la Mort combatu ...

(I.I.I)

La Ceppède déplace toutefois le arma virumque virgilien en parlant des "amours". Son épopée met en scène un héros dont les armes sont l'amour, un amant parfait, qu'il appelle tour à tour "ce divin amant"(I.II.XIX), "ce généreux Amant"(I.III.LXXIV). On comprend, dès lors, que les Théorèmes soient à la fois une épopée et un canzoniere pétrarquiste. Ils reprennent tous les procédés d'un genre surabondamment illustré au $16^{\mathrm{e}}$ siècle: métaphores, enchaînements thématiques et formels, et jusqu'à des schémas complexes de rimes empruntés aux recueils de Philippe Desportes et qui s'écartent de la structure dite régulière du sonnet français; ${ }^{17}$ puis ils sont centrés sur une figure amoureuse, en l'occurence celle du Christ, qui joue ici le rôle dévolu ailleurs à Laure, Cassandre, Marie, Hélène, Francine, Méline ... C'est toutefois au prix d'une transformation assez paradoxale des données du genre. Dans le canzoniere traditionnel, un amoureux repoussé par la femme qu'il aime éperdument se plaint de sa froideur et de ses dédains, décrit sa beauté, ses gestes, les menus événements dont se tisse une passion: c'est celui qui souffre le martyre d'amour qui occupe la position du narrateur. Dans les Théorèmes, par un retournement décisif, c'est la bien-aimée (l'Église), celle à qui s'adresse cet amour sans borne, qui parle et qui décrit les souffrances de son parfait Amant. Il n'est donc pas exact de dire avec Lance $\mathrm{K}$. Donaldson-Evans que "les Théorèmes sont un canzoniere spirituel au centre duquel se trouve, au lieu du personnage féminin traditionnel, le Christ". ${ }^{18}$ Ici, tout se passe comme si l'amoureux pétrarquiste avait traversé toutes les souffrances et comme si sa bien-aimée, émue à la fin de tant de preuves de l'amour le plus extrême, se laissait toucher, chantait elle-même le martyre de son amant et s'exhortait à répondre dignement à une telle passion. Imaginons Le premier livre des Amours écrit par Cassandre, une Cassandre qui se repentirait de tant de dédains, de tant de froideur, de toutes les souffrances qu'elle a infligées à son amant malheureux. On comprend dès lors que la poésie lyrique des canzonieri se charge de valeurs épiques: le changement de point de vue a pour conséquence une objectivation du propos. Les hauts faits d'amour de l'amant, éternisés dans le passé immobile d'une narration fragmentée, assument l'existence objective de tableaux, ils deviennent le lieu d'une méditation et d'un enseignement, ils prennent valeur d'exempla.

Le caractère épique des Théorèmes est donc l'instrument qui permet à La Ceppède de retourner la topique pétrarquiste de la poésie amoureuse, de la purger de tout érotisme, littéralement de la convertir. ${ }^{19}$ Tout le recueil est, en dernière analyse, une méditation sur l'amour, un renversement de la lyrique amoureuse profane qui entend rendre à l'amour sa valeur première, entrevue 
par le néoplatonisme ficinien, de relation au divin. Aussi, loin de s'atténuer, le sentiment amoureux y prend des formes paroxystiques ou hyperboliques, et le topos pétrarquiste rebattu du martyre de l'amoureux en butte aux dédains de sa belle y reprend une vigueur qu'on ne lui connaissait plus. Parce que le Christ est le parfait Amant, sa Passion reproduit tous les moments du récit dont les recueils pétrarquistes proposent d'innombrables versions fragmentées. Ou, plus exactement, la Passion offre le modèle, l'exemplum parfait de toute passion, laquelle se manifeste essentiellement, comme le sait quiconque a lu des recueils de sonnets amoureux, par des souffrances sans limite. C'est donc tout à fait logiquement que La Ceppède tire du martyre du Christ la preuve qu'il est bel et bien le "parfait Amant":

L'Amour l'a de l'Olympe icy bas fait descendre:

L'amour l'a fait de l'homme endosser le peché:

L'amour luy a des-ja tout son sang fait espandre:

L'amour l'a fait souffrir qu'on ait sur luy craché:

L'Amour à ces haliers a son chef attaché:

L'amour fait que sa Mere à ce bois le void pendre:

L'amour a dans ses mains ces rudes cloux fiché:

L'amour le va tantost dans le sepulchre estendre.

Son amour est si grand, son amour est si fort

Qu'il attaque l'Enfer, qu'il terrasse la mort,

Qu'il arrache à Pluton sa fidele Euridice.

Belle pour qui ce beau meurt en vous bien-aimant

Voyez s'il fut jamais un si cruel supplice,

Voyez s'il fut jamais un si parfait Amant.

(I.III.XX)

Le passage de l'amour profane à l'amour sacré, qui conduit La Ceppède à reprendre les figures de la poésie érotique pour les transposer dans un commentaire des récits évangéliques, prend à ses yeux la valeur d'une restitution. Cette conversion de la poésie la ramène à sa pureté primitive, elle lui rend son caractère sacré, divin. On se souviendra que l'Avant-propos à la France évoque cette splendeur antique d'une Muse vouée à la louange de l'Éternel. Dans cette perspective, l'histoire de la poésie, en particulier son histoire récente, apparaît comme une progressive dégradation:

Toutefois le retour des années l'a veue depuis si defformée, qu'à peine recognoissoit-on plus en elle rien de celeste, n'y d'immortel; si qu'au lieu d'estre toujours la mignonne du tout-Puissant, et de servir à ses offices sacrez, elle estoit devenue la servante des hommes corrompus; et n'estoit plus employée qu'à l'adoration des feintes deitez, qu'à la menteuse flaterie, dont elle endormoit les grands vicieux au chant de leurs fausses louanges; 
qu'au maquerelage des folastres et lascives amours. Et bien que la mollesse et que la corruption de la plus grande partie de ceux qui l'ont possédée, luy eussent laissé croistre si longs les cheveux de l'Idolatrie, du mensonge, et de la volupté, qu'elle en fut très-horrible; si l'avoient-ils fardée si subtilement et si pompeusement parée, que facilement la peu caute jeunesse couroit apres ce masque trompeur, et se laissoit piper à cette amorce aleschante. Jen parle comme experimenté; car dés le plus tendre avril de mon âge, affriandé de ses chatouilleuses mignardises, je la receus comme ma plus delicate delice; mais bien-tost apres (esclairé d'un favorable rayon du Soleil de Justice) je la demasqué, et recognu que celle, qui fut jadis fille du Ciel estoit devenue serve de l'Enfer, et que toutes ses premieres beautez estoient non seulement fanées, ains presque ruinées par l'hideuse laideur des vices. ${ }^{20}$

Ce jugement, La Ceppède n'est évidemment pas seul à le porter à l'époque - qu'il suffise de rappeler La Muse chrestienne de du Bartas, antérieure d'une quarantaine d'années aux Théorèmes. Et il n'est pas seul non plus à proposer une poésie rechristianisée, ni seul enfin à entreprendre la composition d'un recueil de sonnets religieux. Toutefois, je le crois bien seul à entreprendre la rédaction d'un véritable canzoniere chrétien, c'est-à-dire à convertir ce genre poétique qui est, sinon le plus grand de la Renaissance, en tout cas le plus caractéristique, le plus répandu. Transposant la relation entre l'amoureux et sa maîtresse, La Ceppède fait du Christ le parfait Amant et il reprend le réseau des métaphores pétrarquistes, le plus riche de toute la lyrique de la Renaissance, que des centaines de recueils avaient surélaboré. Ce véritable coup de génie lui permet aussi de retrouver tout un autre réseau de figures, d'origine théologique celles-là, ce qu'il se fait fort de prouver dans ses abondantes notes qui présentent le Christ et l'Église comme des époux allégoriques:

De l'os tiré du flanc de cet Adam nouveau

L'Eternel a formé son espouse constante...

(I.I.XXII)

D'un seul coup, il assure la parfaite orthodoxie de son oeuvre, il renoue avec l'exégèse traditionnelle ${ }^{21}$ et christianise le lyrisme amoureux de son temps. Ce lyrisme, il n'entend pas l'abolir ou le nier, mais au contraire lui rendre sa splendeur première.

Je prins resolution de l'arrester encore avecque moy, et de tenter si par ce mesme moyen je pourroy restaurer ses anciennes beautez, la rendre d'esclave libre, de Payenne Israelite, et tirer encor qu'elle un Iezrahel. Or pour luy descoudre ses mondains habits (ou plutost habitudes), pour luy raire ses cheveux idolatres, menteurs et lascifs; j'advisay qu'on ne pouvoit mettre en œuvre un outil plus utile que le rasoir à double tranchant de la profonde meditation de la Passion et mort de nostre son Sauveur Jesus-Christ: 
en quoy certes je ne me trompay nullement; car des lors qu'elle se sentit seulement toucher de cest heureux cautere elle mesme deschira ses vieux habillemens, et s'arracha sa Medusine perruque.22

C'est pourquoi on ne peut pas considérer La Ceppède comme un poète antipétrarquiste, comme un négateur des poètes de la Pléiade et de leurs émules. Il faut plutôt voir en lui un écrivain qui reprend le projet de ses prédécesseurs "profanes", qui se place à l'intérieur du même langage, et qui entreprend de parfaire l'œuvre qu'ils n'ont pas menée à terme. Convertissant le lyrisme pétrarquiste, il ne se propose pas de l'abolir mais de l'accomplir.

\section{Université de Montréal}

\section{Notes}

1 Par exemple: Jean Rousset, Anthologie de la poésie baroque française (Paris: Colin, 1961); Marcel Raymond, La Poésie française et le maniérisme (Genève: Droz, 1971); Gisèle Mathieu-Castellani, Éros baroque (Paris: U.G.É., "10/18", 1979).

2 Frédéric Lachèvre, Recueils collectifs de poésie au XVIe siècle (Paris: Champion, 1922); Bibliographie des recueils collectifs de poésie de 1597 à 1700, 4 vol. (Paris: Leclerc, 1901-1905).

3 Cf. John McClelland, "Lieu commun et poésie à la Renaissance”, Études françaises 13.1-2 (avril 1977), pp. 53-70.

4 Les Epithetes de M. de La Porte, parisien. Livre non seulement utile à ceux qui font profession de la Poesie, mais fort propre aussi pour illustrer toute autre composition Françoise. Avec briesves annotations sur les noms et dictions difficiles. (Paris: Chez Gabriel Buon au clos Bruneau à l'image de Sainct Claude, 1571; réimpression: Genève: Slatkine Reprints, 1973).

5 Cf. Bernard Beugnot, "Florilèges et Polyantheae: diffusion et statut du lieu commun à l'époque classique", Études françaises 13.1-2 (avril 1977), pp. 119-142.

6 Textes dans: Gisèle Mathieu-Castellani, Éros baroque, pp. 140 (Scalion de Virbluneau), 141 (Pierre Cornu), 138 (Claude de Pontoux), 143 (Joachim Blanchon); Marcel Raymond, La Poésie française et le maniérisme, pp. 116 (François Ménard), 111 (Laugier de Porchères), 112 (Pierre de Deimier), 91 (Philippe Desportes); Jean Rousset, Anthologie de la poésie baroque française, t. I, p. 126 (Sigogne). Jean de La Ceppède, Les Théorèmes sur le Sacré Mystère de Nostre Rédemption (Toulouse: 1613-1622; reproduction avec préface de Jean Rousset: Genève: Droz, 1966), I.I.LI et I.II.LXX. Les références aux poèmes de La Ceppède seront désormais indiquées entre parenthèses dans le texte.

$7 \mathrm{Cf}$. Jacques Bailbé, "Le thème de la vieille femme dans la poésie satirique du $16^{\mathrm{e}}$ et du début du $17^{\text {e }}$ siècles", B.H.R. XXVI (1964), pp. 98-119; Robert Melançon, "Le Pétrarquisme travesti de Sigogne", Études françaises 13.1-2 (avril 1977), pp. 71-88.

8 Cf. Lance K. Donaldson-Evans, Poésie et méditation chez Jean de La Ceppède (Genève: Droz, 1969), pp. 185-193.

9 Jean de La Ceppède, Les Théorèmes (1613), pp. 10-11.

10 La Ceppède emploie en effet le mot d" "annotations" plutôt que celui de "commentaires", mais on peut penser qu'il ne désigne ainsi que les notes proprement dites qui s'attachent à chaque sonnet. "Commentaires" désigne donc, outre les annotations proprement dites, tout l'appareil de prose qui encadre et soutient les poèmes: l'avant-propos, l'argument de chaque livre, la table des sonnets, l'index des auteurs, la "Table alphabétique très-ample des poincts principaux, et matières plus remarquables traitées en cet œuvre de Theoremes, sur le sacré mystere de nostre Redemption". Sur le genre du commentaire, cf. Jean Céard, "Les transformations du genre du commentaire", dans L'Automne de la Renaissance (Paris: Vrin, 1981), pp. 101-115. 
11 Je n'ai pu consulter l'ouvrage d'Yvette Quenot, Les lectures de La Ceppède (Genève: Droz, "Travaux d'humanisme et Renaissance", 1986), qui n'était pas paru lorsque j’ai rédigé cette communication.

12 Cf. Jean Jehasse, La Renaissance de la critique - l'essor de l'Humanisme érudit de 1560 d 1614 (Saint-Étienne: publications de l'Université de Saint-Étienne, 1976).

13 Cf. Terence C. Cave, Devotional Poetry in France (London: Cambridge University Press, 1969).

14 Ronsard, Abbregé de l'Art poetique François, dans Euvres complètes, éd. Gustave Cohen (Paris: Gallimard, "Bibliothèque de la Pléiade", 1958), t. II, p. 996.

15 La Ceppède, op. cit., note 9, p. 5.

16 Idem, pp. 4-5.

17 Cf. Lance K. Donaldson-Evans, op. cit., note 8, pp. 16-17.

18 Idem, p. 133.

19 Cf. Paul Chilton, The Poetry of Jean de La Ceppedde (Oxford: Oxford University Press, 1977), pp. 89-106, pour une analyse remarquable de cette conversion des Muses.

20 La Ceppède, op.cit., note 9, pp. 5-6.

21 Cf. Paul Chilton, op. cit., note 19, pp. 65-88.

22 La Ceppède, op. cit., note 9, p. 6. 